\title{
Polycystic ovary syndrome: insight into pathogenesis and a common association with insulin resistance
}

\author{
Authors: Thomas M Barber, ${ }^{A}$ George K Dimitriadis, ${ }^{B}$ Avgi Andreou ${ }^{C}$ and Stephen Franks ${ }^{D}$
}

Polycystic ovary syndrome (PCOS) is a common condition that typically develops in reproductive-age women. The cardinal clinical and biochemical characteristics of PCOS include reproductive dysfunction and hyperandrogenic features. PCOS is also strongly associated with obesity based on data from epidemiological and genetic studies. Accordingly, PCOS often becomes manifest in those women who carry a genetic predisposition to its development, and who also gain weight. The role of weight gain and obesity in the development of PCOS is mediated at least in part, through worsening of insulin resistance. Compensatory hyperinsulinaemia that develops in this context disrupts ovarian function, with enhanced androgen production and arrest of ovarian follicular development. Insulin resistance also contributes to the strong association of PCOS with adverse metabolic risk, including dysglycaemia, dyslipidaemia and fatty liver. Conversely, modest weight loss of just $5 \%$ body weight with improvement in insulin sensitivity, frequently results in clinically meaningful improvements in hyperandrogenic, reproductive and metabolic features. Future developments of novel therapies for obese women with PCOS should focus on promotion of weight loss and improvement in insulin sensitivity. In this context, therapies that complement lifestyle changes such as dietary modification and exercise, particularly during the maintenance phase of weight loss are important. Putative novel targets for therapy in PCOS include human brown adipose tissue.

KEYWORDS: PCOS, insulin resistance, metabolic dysfunction, obesity

Authors: ${ }^{A}$ associate professor and honorary consultant endocrinologist, Division of Biomedical Sciences, Warwick Medical School, Coventry, UK, and Warwickshire Institute for the Study of Diabetes, Endocrinology and Metabolism, University Hospitals Coventry and Warwickshire, Coventry, UK; ${ }^{\mathrm{B}}$ Clinical research fellow, Division of Biomedical Sciences, Warwick Medical School, Coventry, UK, and Warwickshire Institute for the Study of Diabetes, Endocrinology and Metabolism, University Hospitals Coventry and Warwickshire, Coventry, UK; ${ }^{C}$ clinical research fellow, Warwickshire Institute for the Study of Diabetes, Endocrinology and Metabolism, University Hospitals Coventry and Warwickshire, Coventry, UK; ${ }^{D}$ professor of reproductive endocrinology, Institute of Reproductive and Developmental Biology, Imperial College, London, UK

\section{What is polycystic ovary syndrome?}

Polycystic ovary syndrome (PCOS) is a common endocrine condition that affects women of reproductive age. Depending on the population studied, the prevalence of PCOS in premenopausal women has been estimated between 6 and 10\%. The clinical and biochemical characteristics of PCOS can be grouped into two main categories: (i) reproductive features that include oligo-amenorrhea (irregular, infrequent or absent menses), and (ii) hyperandrogenic features that include hirsutism, acne, androgenic alopecia and hyperandrogenaemia (raised serum levels of androgens such as testosterone). ${ }^{2}$ In addition to these clinicobiochemical features, polycystic ovarian (PCO) morphology shown on ultrasound scan is a common radiological feature of PCOS. The three cardinal characteristics outlined here (reproductive, hyperandrogenic and PCO morphological features) form the basis for the internationally agreed consensus diagnostic criteria for PCOS known as the 'Rotterdam Criteria. ${ }^{3}$ On this basis, women with at least two of these key characteristics, following exclusion of other conditions that can masquerade as PCOS (including congenital adrenal hyperplasia and ovarian and adrenal androgen-secreting tumours), can be diagnosed with PCOS. It is important, however, to emphasise that PCO morphology is itself a common ultrasonic feature in $20 \%$ of reproductive-age women, most of whom have minimal or no symptoms of the syndrome; ${ }^{4}$ therefore, most women with PCO morphology do not have PCOS. Furthermore, PCOS is also a condition that associates with obesity, insulin resistance and cardiometabolic risk, although these features do not form part of the diagnostic criteria for PCOS. ${ }^{3}$

The association between PCOS and obesity is clear from epidemiological studies that reveal the majority of women (38-88\%) with PCOS to be either overweight or obese. ${ }^{2}$ These epidemiological data are further strengthened by observations which show that even modest weight loss of just $5 \%$ in obese women with PCOS can translate into improved reproductive and hyperandrogenic clinicobiochemical features, including hirsutism, menstrual cyclicity and fertility. ${ }^{5}$ Further evidence to corroborate the association between PCOS and obesity were provided by our own group, revealing association of variants within the FTO gene and susceptibility for development of PCOS, through effects on fat mass. ${ }^{6}$

The association of obesity with PCOS is thought to be important for its development, given that PCOS often becomes clinically manifest in women who gain weight and 
are genetically predisposed to its development. Furthermore, as previously outlined, the clinical features of PCOS often improve with weight loss. However, it is also clear that, independent of obesity and fat mass, PCOS is inherently associated with metabolic aberrations that include insulin resistance, dyslipidaemia and non-alcoholic fatty liver disease. ${ }^{7}$ Furthermore, women with PCOS are at a much higher risk than weight-matched control women for developing dysglycaemia: among women with PCOS, $10 \%$ have type-2 diabetes mellitus and $30-40 \%$ develop early-onset impaired glucose tolerance. ${ }^{8,9}$ Metabolic syndrome is also more common in women with PCOS compared with BMI-comparable control women. Based on National Cholesterol Education Program Adult Treatment Panel III criteria, 34-46\% of US-based Caucasian women with PCOS have metabolic syndrome. ${ }^{8}$ Further contributing to this dysmetabolic onslaught is the common association of PCOS with obstructive sleep apnoea (OSA), the risk of OSA being 5-10-fold higher in PCOS than in BMI-matched control women. ${ }^{9}$ OSA is a condition which itself also independently associates with insulin resistance that, in turn, further worsens metabolic dysfunction in PCOS. ${ }^{9}$

To summarise, obese women with PCOS typically manifest a metabolic 'double-whammy' from conspiring metabolic aberrations associated with obesity and PCOS. ${ }^{2}$ Insulin resistance is closely associated with the metabolic aberrations that characterise PCOS. Given that insulin resistance is important in the development of PCOS, it is important to gain insight into the pathogenic mechanisms implicated.

\section{Insulin resistance in the pathogenesis of PCOS}

It is estimated that between 50 and $90 \%$ of women with PCOS manifest insulin resistance. ${ }^{10}$ As a compensatory response to insulin resistance, hyperinsulinaemia develops which in turn interacts synergistically with luteinising hormone (LH) as a co-gonadotrophin within ovarian theca cells. ${ }^{11}$ Resultant activation of CYP17 (encoding P450c17 $\alpha$, a key enzyme in ovarian androgen biosynthesis) in turn enhances the generation and release of androgens. ${ }^{12}$ Within the ovary, insulin also promotes arrest of pre-antral follicle development. ${ }^{13}$ Hyperinsulinaemia also has other extra-ovarian pleiotropic effects that include enhancement of LH pulse amplitude (demonstrated in rodent models), ${ }^{14}$ stimulation of adrenal P450c17 $\alpha$ activity, ${ }^{12}$ and suppression of hepatic sex hormone binding globulin production. ${ }^{15}$ An overview of peripheral insulin resistance and its role in the pathogenesis of PCOS is shown in Fig 1.

The role of insulin resistance in the pathogenesis of PCOS is supported by observations that improving insulin sensitivity in this condition (through weight loss or drug therapy) improves reproductive, hyperandrogenic and metabolic features. ${ }^{2}$ The converse is also true: weight gain (with worsening insulin resistance) in those women who are genetically predisposed to the development of PCOS often results in them manifesting clinical features of this condition. ${ }^{2}$ There is a paradox though in reconciling insulin resistance with the deleterious effects of hyperinsulinaemia, given that both insulin resistance and hyperinsulinaemia are important for the development of PCOS. This apparent paradox is resolved when one considers the molecular pathways involved. Following interaction with its receptor, intracellular effects of insulin follow two main pathways via: (i) phosphatidylinositol 3-kinase (PI3-kinase) and (ii) mitogen-activated protein kinase (MAP kinase). Each of these pathways mediates disparate cellular effects of insulin stimulation. The PI3-kinase pathway mediates metabolic effects (including glucose disposal into skeletal muscle) and the MAP kinase pathway mediates cell growth and steroidogenic effects. ${ }^{16}$ In PCOS, it appears that only the PI3-kinase pathway is dysfunctional, with the MAP kinase pathway functioning normally. ${ }^{2}$ The result is divergent cellular responses to insulin, with resistance to its metabolic effects and concurrent enhancement of steroidogenesis manifesting as

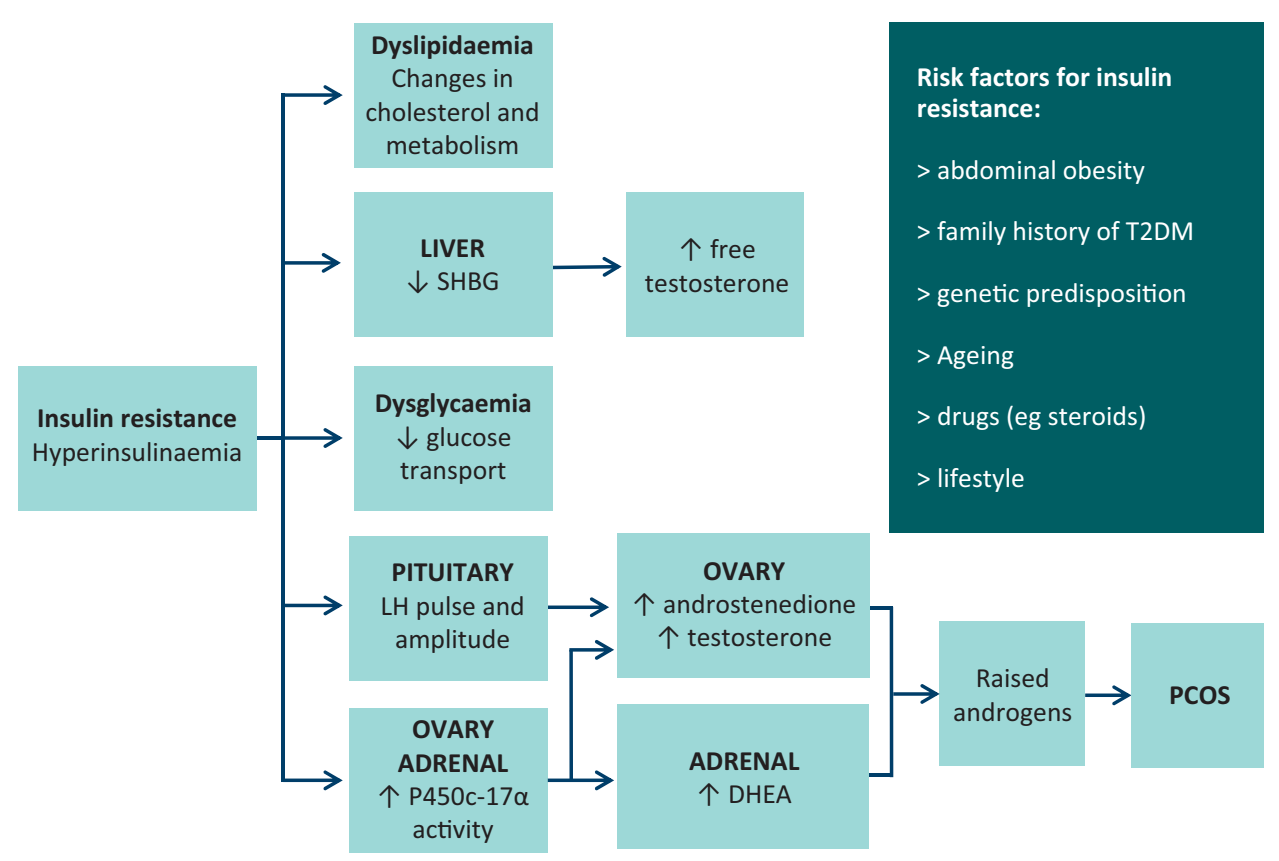

Fig 1. Overview of peripheral insulin resistance in PCOS. DHEA = dehydroepiandrosterone; $\mathrm{LH}=$ luteinising hormone; $\mathrm{PCOS}=$ polycystic ovary syndrome; SHBG = sex hormone binding globulin; T2DM = type-2 diabetes mellitus. 
metabolic dysfunction, hyperandrogenaemia and reproductive dysfunction. ${ }^{17}$

The intact MAP kinase receptor pathway is important in the mechanism by which insulin resistance influences development of hyperandrogenaemia in PCOS. In a comparison between urinary steroid profiles in women with PCOS $(n=178)$ and BMI-matched control women $(n=100)$, our own group demonstrated enhanced $5 \alpha$ reductase activity associated with PCOS. ${ }^{18}$ Resulting enhanced conversion of testosterone to the more potent androgen, $5 \alpha$-dihydrotestosterone likely contributes to the hyperandrogenism of PCOS. A further effect of enhanced $5 \alpha$ reductase activity is the breakdown of cortisol with reduced negative feedback at the pituitary. Consequently, the hypothalamo-pituitary adrenal axis becomes overactive in PCOS, thereby further stimulating adrenal androgen production. $^{18}$

Further insight into the development of insulin resistance in PCOS can be gained from exploring its occurrence across different phenotypic subgroups and associations with visceral fat depots. These two topics will be discussed in the following subsections.

\section{Metabolic heterogeneity among phenotypic subgroups of PCOS}

Use of the 'Rotterdam diagnostic criteria' for PCOS has led to the emergence of four distinct phenotypic subgroups. ${ }^{3}$ In this section, we refer to these as 'HO', 'PHO', 'PH' and 'PO' with ' $\mathrm{H}$ ' representing hyperandrogenic features, 'O' representing oligo-amenorrhea and ' $\mathrm{P}$ ' representing $\mathrm{PCO}$ morphology. Our own group published data from UK-based Caucasian women with Rotterdam-defined PCOS $(\mathrm{n}=309)$ in comparison with control women $(\mathrm{n}=76) .{ }^{3,19}$ In this study, we showed that insulin resistance is predominantly a feature of the 'PHO' sub group and that metabolic syndrome is also common in this subgroup. ${ }^{19}$ This disparity of metabolic dysfunction between phenotypic subgroups, with metabolic equivalence versus BMI-matched control women for ' $\mathrm{PH}$ ' and 'PO' subgroups, has subsequently been verified in other studies on diverse populations. ${ }^{20,21}$ Current literature supports the notion of metabolic heterogeneity between Rotterdam-defined phenotypic subgroups of PCOS, ${ }^{3}$ with insulin resistance and metabolic dysfunction largely confined to the subgroup of majority who manifest reproductive and hyperandrogenic features with PCO morphology.

Current literature on metabolic heterogeneity in PCOS focuses on cross-sectional data. There is a need for long-term prospective studies in this field, to track potential migration between phenotypic subgroups over time, and to explore the impact of cumulative phenotypic subgroup status and its associated cardiometabolic risk profile on future cardiovascular outcomes. Such a study would provide insight into predictors of future cardiovascular events and facilitate development of strategies to improve the cardiovascular fate of obese women with PCOS.

\section{Role of visceral fat as a contributor towards insulin resistance in $\mathrm{PCOS}$}

It is important to explore potential mechanisms that underlie the development of insulin resistance in PCOS. ${ }^{2}$ One possible factor relates to body fat distribution, given the well established association between visceral adipose tissue and insulin resistance. ${ }^{22,23}$ Unfortunately, many previous studies on fat distribution in PCOS utilised techniques such as ultrasound and lipometer that suffer from limitations of operatordependence and inadequate image resolution. ${ }^{23}$ Our own group utilised an magnetic resonance (MR)-based imaging technique for assessment and comparison of fat distribution among 22 obese BMI- and fat mass-matched pairs of PCOS cases and controls. Cross-sectional areas of fat depots from axial MR images taken at anatomically pre-defined sites were measured and compared between groups. ${ }^{24}$ Although disparate with regard to insulin sensitivity, there were no significant differences in fat depots, including visceral fat between the groups of women with PCOS and BMI-matched control women. ${ }^{24}$ Data from our study ${ }^{24}$ were subsequently corroborated by a further MR-based study on abdominal fat depots in 31 age- and BMI-matched pairs of PCOS cases and controls that also demonstrated equivalence of fat depot volumes and distributions between the two groups. ${ }^{25}$

To conclude, women with PCOS manifest global adiposity. Based on our own data ${ }^{23,24}$ and from those of a large dualenergy X-ray absorptiometry-based study on PCOS and weightmatched control women, ${ }^{26}$ abdominal fat mass in women is proportional to total fat mass regardless of PCOS status. This is what one would expect and helps to explain worsening of insulin resistance in women who gain weight. An important conclusion from the data outlined here is that insulin resistance in PCOS cannot be explained by preponderance of visceral fat in this condition. It remains possible that aberrations of ectopic fat distribution may pertain in PCOS that may contribute to insulin resistance and this should be a focus for future research. ${ }^{23}$

\section{Improvement of insulin resistance in the management of PCOS}

Given the importance of insulin resistance in the development of PCOS, it is perhaps not surprising that weight loss, and the consequent improvement in insulin sensitivity, remains the most effective treatment strategy for women with PCOS. ${ }^{2}$ Achieving and maintaining weight loss however is challenging. Established strategies of dietary modification and increased exercise are the mainstay, given the current lack of available therapies for weight loss. ${ }^{2}$ Bariatric surgery can also play a role, although the relative cost and availability of this procedure precludes its widespread adoption. ${ }^{27}$

There is a need to develop effective weight-loss therapies, to improve outcome in PCOS and other weight-related conditions. A promising target for such a novel therapy is brown adipose tissue (BAT). Recent observations of active BAT in adult humans have transformed this field. ${ }^{28}$ BAT uncouples oxidative phosphorylation and in the process releases stored caloric energy as heat. ${ }^{28}$ The therapeutic potential of BAT as an antiglycaemic, anti-lipidaemic and weight loss inducing 'metabolic panacea' is exemplified by calculations that just a sugar-cube volume of active BAT during a year, would burn its way through between 3 and $4 \mathrm{~kg}$ of white adipose tissue. ${ }^{29}$ There is much research needed before BAT-related therapies become available. One important consideration is the quantification of BAT, to provide data on widespread applicability of 
BAT-related therapies and to act as a biomarker in assessment of response to such therapies. Our own group has published the first proof of concept paper to image BAT in a living human adult using an MR-technique and verification with histology and immunohistochemistry. ${ }^{30}$ The next stage is to develop this technique as a radio-quantifier for assessment of BAT content in humans.

It is important that BAT-related and other novel therapies that promote weight loss are developed, to complement lifestyle measures that include dietary modification and exercise. Such novel therapies would be particularly beneficial in the maintenance of weight loss, which is notoriously difficult to achieve. Future research should also focus on development of novel therapeutics that improve insulin sensitivity in women with PCOS, therapies that would also be expected to improve the clinical and biochemical manifestations of PCOS.

\section{Concluding remarks}

A major challenge in research into pathogenesis of PCOS is to untangle the complex interlinks between obesity, insulin resistance and the development of PCOS. Our understanding of this complex multi-directional entangled web of interactions is incomplete. Unfortunately, PCOS suffers from much misconception among the general public and healthcare professionals. Some of this misconception stems from nomenclature: the term 'PCOS' belies its true importance as a condition that may have a profound adverse effect on physical and psychological wellbeing. It is a condition that accounts for the commonest cause of infertility in women, causes much psychosocial distress and anxiety from the 'cosmetic' concerns of hirsutism and acne, and is associated with substantial cardiometabolic risk.

The role of insulin resistance in the development of PCOS has been discussed in this brief review. Potential contributors to insulin sensitivity, including fat distribution and phenotypic subgroup have been outlined. Strategies for future developments of therapies for losing weight and maintaining weight loss have been proposed. The prevalence of PCOS is such that every healthcare professional is likely to encounter this condition. The ever burgeoning obesity epidemic will ensure that the prevalence of PCOS continues to increase. To mitigate the impact of obesity on the development of PCOS, it is important to take action now to facilitate weight loss in our obese patients through existing strategies, and to share a vision of the future where development and administration of novel weight-loss strategies complement lifestyle measures and facilitate effective and safe maintenance of weight loss. Through creating a future healthcare environment that encourages maintenance of weight loss, we will limit the impact of obesity and its associated insulin resistance on the development of PCOS and other weight-related conditions.

\section{Note}

This article was originally published in the 2015 Clinical Medicine supplement Horizons in Medicine 27. All articles in this supplement are available at www.clinmed.rcpjournal.org/content//15/Suppl_6

\section{References}

1 Asuncion M, Calvo RM, San Millan JL et al. A prospective study of the prevalence of the polycystic ovary syndrome in unselected Caucasian women from Spain. J Clin Endocrinol Metab 2000;85:2434-8.

2 Barber TM, McCarthy MI, Wass JA, Franks S. Obesity and polycystic ovary syndrome. Clin Endocrinol (Oxf) 2006;65:137-45.

3 The Rotterdam ESHRE/ASRM-sponsored PCOS consensus workshop group. Revised 2003 consensus on diagnostic criteria and long-term health risks related to polycystic ovary syndrome (PCOS). Hum Reprod 2004;19:41-7.

4 Polson DW, Adams J, Wadsworth J, Franks S. Polycystic ovaries - a common finding in normal women. Lancet 1988;1:870-2.

5 Holte J, Bergh T, Berne C, Wide L, Lithell H. Restored insulin sensitivity but persistently increased early insulin secretion after weight loss in obese women with polycystic ovary syndrome. J Clin Endocrinol Metab 1995;80:2586-93.

6 Barber TM, Bennett AJ, Groves CJ et al. Association of variants in the fat mass and obesity associated (FTO) gene with polycystic ovary syndrome. Diabetologia 2008;51:1153-8.

7 Ramezani-Binabaj M, Motalebi $\mathrm{M}$ et al. Are women with polycystic ovarian syndrome at a high risk of non-alcoholic fatty liver disease; a meta-analysis. Hepat Mon 2014;14:e23235.

8 Barber TM, McCarthy MI, Franks S, Wass JA. Metabolic syndrome in polycystic ovary syndrome. Endokrynol Pol 2007;58:34-41.

9 Ehrmann DA. Metabolic dysfunction in PCOS: relationship to obstructive sleep apnea. Steroids 2012;77:290-4.

10 Venkatesan AM, Dunaif A, Corbould A. Insulin resistance in polycystic ovary syndrome: progress and paradoxes. Recent Prog Horm Res 2001;56:295-308.

11 Franks S, Mason H, White D, Willis D. Mechanisms of anovulation in polycystic ovary syndrome. Steroids 1997;62:728.

12 Morin-Papunen LC, Vauhkonen I et al. Insulin sensitivity, insulin secretion, and metabolic and hormonal parameters in healthy women and women with polycystic ovarian syndrome. Hum Reprod 2000;15:1266-74.

13 Willis DS, Watson H, Mason HD et al. Premature response to luteinizing hormone of granulosa cells from anovulatory women with polycystic ovary syndrome: relevance to mechanism of anovulation. J Clin Endocrinol Metab 1998;83:3984-91.

14 Dunaif A. Insulin resistance and the polycystic ovary syndrome: mechanism and implications for pathogenesis. Endocr Rev 1997; 18:774-800.

15 Yki-Jarvinen H, Makimattila S, Utriainen T, Rutanen EM. Portal insulin concentrations rather than insulin sensitivity regulate serum sex hormone-binding globulin and insulin-like growth factor binding protein 1 in vivo. J Clin Endocrinol Metab 1995;80:3227-32.

16 Cusi K, Maezono K, Osman A et al. Insulin resistance differentially affects the PI 3-kinase- and MAP kinase-mediated signaling in human muscle. J Clin Invest 2000;105:311-20.

17 Rice S, Christoforidis N, Gadd C et al. Impaired insulin-dependent glucose metabolism in granulosa-lutein cells from anovulatory women with polycystic ovaries. Hum Reprod 2005;20:373-81.

18 Vassiliadi DA, Barber TM, Hughes BA et al. Increased 5 alphareductase activity and adrenocortical drive in women with polycystic ovary syndrome. J Clin Endocrinol Metab 2009; 94:3558-66.

19 Barber TM, Wass JA, McCarthy MI, Franks S. Metabolic characteristics of women with polycystic ovaries and oligo-amenorrhoea but normal androgen levels: implications for the management of polycystic ovary syndrome. Clin Endocrinol (Oxf) 2007;66:513-7.

20 Vaggopoulos V, Trakakis E, Chrelias C et al. Comparing classic and newer phenotypes in Greek PCOS women: the prevalence of metabolic syndrome and their association with insulin resistance. J Endocrinol Invest 2013;36:478-84 
21 Zhang HY, Zhu FF, Xiong J et al. Characteristics of different phenotypes of polycystic ovary syndrome based on the Rotterdam criteria in a large-scale Chinese population. BJOG 2009;116:1633-9.

22 Zhuang XF, Zhao MM, Weng CL, Sun NL. Adipocytokines: a bridge connecting obesity and insulin resistance. Med Hypotheses 2009;73:981-5.

23 Barber TM, Franks S. Adipocyte biology in polycystic ovary syndrome. Mol Cell Endocrinol 2013;373:68-76.

24 Barber TM, Golding SJ, Alvey C et al. Global adiposity rather than abnormal regional fat distribution characterises women with polycystic ovary syndrome. J Clin Endocrinol Metab 2008;93:9991004 .

25 Manneras-Holm L, Leonhardt H, Kullberg J et al. Adipose tissue has aberrant morphology and function in PCOS: enlarged adipocytes and low serum adiponectin, but not circulating sex steroids, are strongly associated with insulin resistance. J Clin Endocrinol Metab 2011;96:E304-11.

26 Carmina E, Bucchieri S, Esposito A et al. Abdominal fat quantity and distribution in women with polycystic ovary syndrome and extent of its relation to insulin resistance. J Clin Endocrinol Metab 2007;92:2500-5.
27 Escobar-Morreale HF. Surgical management of metabolic dysfunction in PCOS. Steroids 2012;77:312-6.

28 Cypess AM, Lehman S, Williams G et al. Identification and importance of brown adipose tissue in adult humans. $N$ Engl J Med 2009;360:1509-17.

29 Virtanen KA, Lidell ME, Orava J et al. Functional brown adipose tissue in healthy adults. N Engl J Med 2009;360:1518-25.

30 Reddy NL, Jones TA, Wayte SC et al. Identification of brown adipose tissue using MR imaging in a human adult with histological and immunohistochemical confirmation. J Clin Endocrinol Metab 2014;99:E117-21.

\section{Address for correspondence: Dr TM Barber, Division of} Biomedical Sciences, Warwick Medical School, University of Warwick, Clinical Sciences Research Laboratories, University Hospitals Coventry and Warwickshire, Clifford Bridge Road, Coventry CV2 2DX, UK.

Email: t.barber@warwick.ac.uk



